\title{
SIMULATIONS OF THE LONGITUDINAL INSTABILITY IN THE NEW SLC DAMPING RINGS*
}

\author{
K.L.F. Bane and K. Cide ${ }^{\circ}$
}

Stanford Linear Accelerator Center, Stanford University, Stanford, CA 94309 ISA

\section{INTRODUCTION}

In 1992 a longitudinal, single bunch instability was observed in the SLC damping rings.[1] Beyond a threshold current of $3 \times 10^{10} \mathrm{a}$ "saw-tooth" variation in bunch length and energy spread was observed, a phenomenon that made it practically impossible to operate the SLC collicer above threshold. For the 1994 run a new, low-impedance vacuum chamber was ingtalled in both damping rings both to alleviate this problem and to shorten the bunch length. According to recent measurements the bunch length has indeed become shorter, but the "saw-tooth" instability is still seen, now beginning at currents of $1.5-2.0 \times 10^{10} .[2]$ Fortunately, it appears to be benign and does not seem to limit SLC performance.

In an earlier paper we inves.igated the single bunch behavior of the SLC damping rings with the old vacuum chamber using time domain tracking and a Vlasov equation approach.[3] When compared to measurements we found: good agreement in the average values of bunch length, energy spread, and synchronous phase shift as functions of eurrent; a $30 \%$ discrepancy in threshold current; in agreement, a mode with frequency near 2.5 times the synchrotion frequency (the so-called "sextupolen" mode) as signature of the instability and the slope of the mode frequency as function of current.' In the present paper we repeat the exercise of the earlier paper but with a new wakefield. The impedance which used to be inductive has become resistive, leading to lifferent phenomena.

In a recent paper the instability in a purely resistive ring is analyzed using a Vlasov equation approach. [5] it is demonstrated that such an instability is a weak instability, with a growth rate proportional to intensity squared, and one that can be described as the coupling of two quadrupole modes with different radial mode numbers. We will compare our sesults with this paper. For related papers, see also Refs. [6,7].

\section{THE WAKEFIELD}

In the vecuum chamber upgrade of the SLC damping inge primarily small objects that are inductive at nominal bunch lengths $(\sim \mathbf{5} \mathrm{mm})$ were removed or modified, $[2,8]$ changing the character of the rings from inductive to resislive. $\lambda_{s}$ before, we have attempted to find an approximate Gieen function wakefield $W(z)$ for the new ring using the time-domain parts of the MAFIA family of computer programs, [9] taking as driving hunch a short, gaussian bunch with rms length of $1 \mathrm{~mm}$. To make it causal, the part in front of bunch center $(z<0)$ was reffected and added to the back (see Fig. 1), a transformation that preserves the real part of the impedance. We expect to be able to find beam instabilities down to wavelengths of about $1 \mathrm{~cm}$.

-Work supported by Department of Energy contract DEAC03-76SF00515.

ofrom KEK.

IIn Ref. 3 it was stated that the measured slope was three times smaller than the calculations. However, subsequent, more accurate measurements are in agreement with the calculations. [4]

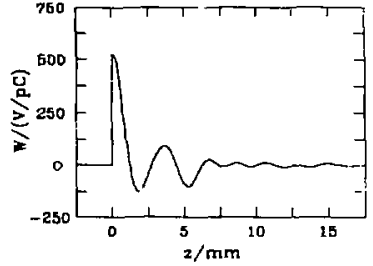

Fig. 1. The wakefield used for the simulations.

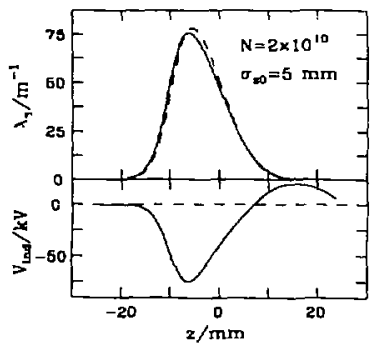

Fig. 2. A potential well example.

We have had difficulty obtaining an accurate wakefield, and we are not very satisfied with what we have: it should be considered only preliminary. As we have removed the grosser, cylindrically symmetric objects $\left(45^{\circ}\right.$ transitions, masks, etc.) we are left with a machine dorninated by objects for which it is difficule to obtain an accurate wake function, such as, for example. the beam position monitors. Also, difficult vacuum chamber objerts that could previously be ignored, such as the septum chamber, may now be important. In spite of these misgivings, and even though (as we will see) the results do not agree with the SLC measurements as well as before. we foel thal. this wakefield is stili useful for studying the basic character of the damping ring current dependent behavior.

The induced voltage on any turn is given by

$$
V_{\text {ind } d z}(z)=-e N \int_{-\infty}^{2} W\left(z-z^{\prime}\right) \lambda z\left(z^{\prime}\right) d z^{\prime}
$$

with $N$ the bunch population and $\lambda_{4}(z)$ the longitudiual charge distribution. To see that it is resistive in character at typical bunch lengths we plot in Fig. $2 \lambda_{z}$ and $l_{i n d}$ for $N=2 \times 10^{10}$ and nominal length $\sigma_{20}=5 \mathrm{~mm}$, the solution to the Haissinski equation:[10] We note that roughly $V_{\text {unis }}$ can be written as $V_{\text {und }} \approx-e N R c \lambda_{2}$, with $R$ the resistance. a constant. In fact, if we take a pure resistance wilh $R=$ $880 \Omega$, and repeat the potential well calculation, we olit ain almost thie same bunch shape (the dashes in Fig. 2). 


\section{SIMULATIONS}

For tracking we let the beam be represented by $N_{p}$ macro-particles; each particle $\boldsymbol{i}$ has position and energy coordinates $\left(z_{i}, \epsilon_{i}\right)$. The properties of particle $i$ are advanced on each turn according to the equations:[11]

$$
\begin{gathered}
\Delta \epsilon_{i}=-\frac{2 T_{0}}{T_{d}} \epsilon_{i}+2 \sigma_{c 0} \sqrt{\frac{T_{0}}{T_{d}}} r_{i}+V_{r j}^{\prime} z_{i}+V_{\text {ind }}\left(z_{1}\right) \\
\Delta z_{i}=\frac{\alpha c T_{0}}{E_{0}}\left(\epsilon_{i}+\Delta \epsilon_{i}\right) .
\end{gathered}
$$

with $T_{0}$ the revolution period, $\tau_{d}$ the damping time, $\sigma_{t 0}$ the nominal ims energy spread, $V_{r j}^{\prime}$ the slope of the rf voltage (a negative quantity), $\alpha$ the momentum compaction factor, and $E_{0}$ the machine energy; $r_{i}$ is a random number from a normal set with mean 0 and rms 1 . To calculate $\lambda_{2}$ on each turn we bin the macro-particles in $z$.

For the simulations we take $T_{0}=118 \mathrm{~ns}, E_{0}=$ $1.15 \mathrm{GeV}$, if frequency $\nu_{\mathrm{r} f}=714 \mathrm{MHz}, \sigma_{\mathrm{cO}}=0.07 \%$, and $r_{d}=1.7 \mathrm{~ms}$. We choose $V_{r j}=0.8 \mathrm{MV}$, where $\sigma_{z 0}=$ $4.95 \mathrm{~mm}$, and the synchrotron firequency $\nu_{s 0}=99 \mathrm{kHz}$. Therefore $\nu_{0} T_{0}=85$ turns, $r_{d} / T_{0}=14450$ turns. We take $N_{p}=30.000$, and for $\lambda_{z}$ we use 100 bins extending over $10 \sigma_{2}$. We let the program run for 3 damping times.

As a second method of calculation we use a computer program that solves perturbatively the time independent, linearized Vlasov equation, includiug the effects of potential well distortion, looking for unstable modes.[12] Beyond the tireshold current we assume the average energy distribution remains gaussian, with the rms width $\sigma_{t}$ incrcasing to keep the beam just at the threshuld condition.

\section{RESULTS}

\section{The Instability Threshold}

For this wakefield the instability threshold is normally easy to find from the turn-by-turn tracking results. Below threshold the moments of the distributions are well behaved, above thresbold they undergo macroscopic oscillations (see Fig. 3 ). The threshold $N_{\mathrm{sh}} \approx 1.15 \times 10^{10}$.

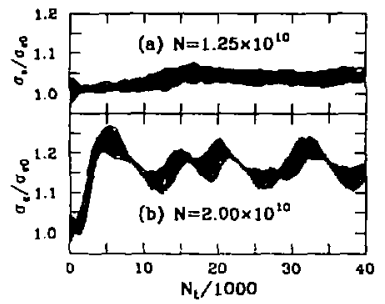

Fig. 3. The turn-by-turn rms energy spread just above threshold (a) and at a higher current. (b)

When we artificially reduce the damping time in tracking we find that the threshold $N_{\text {th }}$ increases significantly (see Fig. 4). Fitling to a power law we find that $N_{t h}$ varies approximately as $\tau_{d}^{-1 / 2}$ (the curve in Fig. 4). This agrees with the weak growth expected in a purely resistive machine, which varies as $\sim e^{a N^{2} t}$, with $a$ a constant and $t$ time.[5] Note that when we repeat the tracking procedurc for the old, inductive vacuum chamber the threshold increases by only $30 \%$ as the damping time is decreased by a factor of 15 . The two instabilities are quite different: the old was a strong instability and the new a weak one

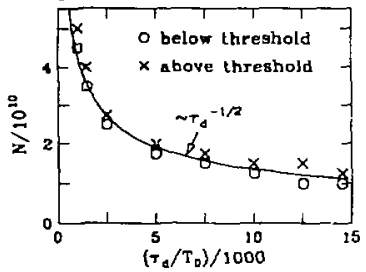

Fig. 4. $N_{\text {in }}$ vs. $\tau_{d}$ obtained by tracking.

According to a formula in Ref. 5 ( $E_{\text {ql. }} 27$ ) we can raise the threshold by $\sim 1 \times 10^{10}$ if we add a pure induclance of $L=2 \mathrm{nH}$ (by adding a term $-e N c^{2} L \lambda_{z}^{\prime}$ to $V_{1 n d}$ ). This is roughly what. we find: and at $N^{*}=4 \times 10^{10} \sigma_{l} / \sigma_{t 0}$ has decreased from 1.5 to 1.1. This suggests that even a small amount of tune spread can damp this instability

\section{Average Bunch Properties}

Above threshold the oscillations in the moments of the distributions obtained by tracking can be large (at $N=3.5 \times 10^{10}$ the variation in $\sigma_{z}$ is $\pm 28 \%$ ) and the pat tern can vary greatly. Il depends sensitively on. for exam. ple, $N_{p}$, which (for practical reasons) it is difficult for us to increase signific antly, while beeping $\tau_{d}$ fixed. However. the average amplitudes appear to be rather insensitive to changes in $N_{p}$. Fig. 5 gives the average values of $\sigma_{i}, \sigma_{l}$, and $\langle z\rangle$ as functions of $N$, and also shows the Vlasov equation solution for comparison. The agreement is quite good. Note that for such a resistive impedance the potential well distortion is small; the bunch lengthening is largely due to the increase in energy spread above threshold. Thus. by adding $2 \mathrm{nH}$ of pure inductance to reduce the energy spread at $4 \times 10^{10}$ the $60 \%$ increase in $\sigma_{\alpha}$ is reduced to $30 \%$

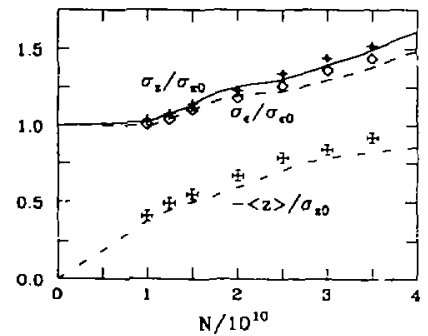

Fig. 5. A verage bunch properties us $N$. Shown are tracking results (plotting symbols) and the Vlasov method results (curves)

Fig. 6 gives contours of phase space of the unstal, Ir mode at $N=2 \times 10^{10}$ as calculated by the Vlasov nemllod Tracking gives a similar result. We see a quadrupole node, with a slight asymmetry, that has heen shifted forward. 


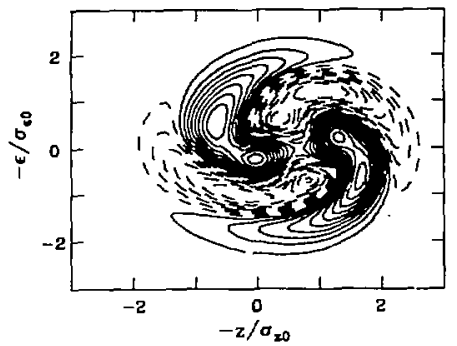

Fig. 6. Mode shape at $N=2 \times 10^{10}$.

\section{The Spectrum}

The mode frequencies as function of $N$, as obtained by the Vlasov method are shown in Fig. 7. A dot represents a stable mode, an ' $X$ ' an unstable mode, with its size proportional to the growth rate. The strongest unstable mode is a quadrupole mode beginning at $N=1 \times 10^{10}$ with $\nu=1.95 \nu, 0$, and then continuing with a glope of $-0.07 v, 0 / 10^{10}$. As was the case for a purely resistive machine the instability can be described as the coupling of two radial modes with the same azimuthal mode number.

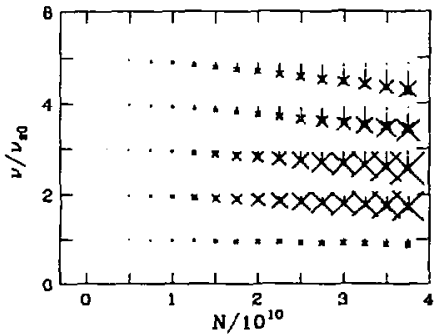

Fig. 7. Modes obt-ined by the Vlasov method.

For the tracking results, by Fourier transforming any of the turn-by-turn moments of the distribution we can obtain the spectrum. Alternatively, we can simulate what a spectrum analyzer does by cajculating[13]

$$
g(\omega)=\mid \sum_{k} e^{i \omega \cdot k T_{0} \bar{\lambda}_{z}(\omega) \mid},
$$

with $k$ the turn number and $\dot{\lambda}_{25}(\omega)$ the Fourier transform of the distribution on the $k^{t h}$ turn. We find sidebands at $1.9 v, 0$, but because of numerical noise we have poor resolution. To give an example with good resolution let us set $N_{p}=150,000$, artificialiy reduce $\tau_{d}$ by a factor of 10 , and consider $N=4 \times 10^{10}$. In Fig. 8 we display the result near a central friquency $\nu_{c}=30 \mathrm{GHz}$. We see sidebands of the revolution irequency separated by $1.785 \nu_{30}$. Note that the sideband amplitudes are not of equal height. In general, potential well distortion tends to result in an asymmetric inode shape; therefore, for frequencies $\nu_{c} \geq c / 2 \pi \sigma_{z}$ we expect the sidebands to be of unequal height

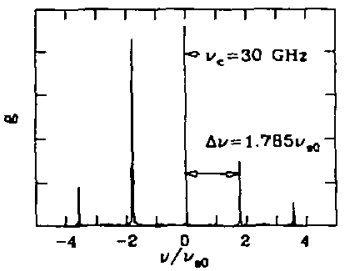

Fig. 8. Spectrum for $N=4 \times 10^{10}, \tau_{d} / T_{0}=1450$.

\section{COMPARISON WITH MEASUREMENTS[2]}

In the measurements the buncli length is smaller and the bcam profile more asymmetric than before, and above threshold a frequency just below $2 v_{s} 0$ is observed, which are consistent with a resistive wakefield and our simulalions. In detail, the agreement is not good unless we asume our wake is missing about $2 \mathrm{nH}(|Z / n|=0.1 \Omega)$ of pure inductance. The measurements give: $N_{t h}=1.5-2.0 \times 10^{10}$, and at $N=4 \times 10^{10} \sigma_{t} / \sigma_{10}=3.15$. and $\sigma_{2} / \sigma_{20}=1.25$ : the calculations including the inductance give: $N_{1 h}=$ $2.0 \times 10^{10}$, and al $N=4 \times 10^{10} \sigma_{1} / \sigma_{10}=1.10$, and $\sigma_{*} / \sigma_{20}=1.30$. The unstable mode frequency at threshold and the slope, given by measurements (calrulations, including $2 \mathrm{nH}): 1.77(1.87) \nu_{\mathrm{s}}$ and $-.06(-.07) \nu_{00} / 10^{10}$.

Finally, how can we understand the reduction of the measured threshold when the damping ring impedance was reduced? In the old, inductive machine there was a strong instability observed at $3 \times 10^{10}$. In an inductive machire there is a large incoherent synchrotron tune spread which will Landau damp weaker instabilities which otherwise might appear at lower currer:i: By removing mostly inductive elemenis, and thereby changing the character of the ring to a resistive one, we have removed this tune spread, and presumably are now able to observe one of these weaker instabilities.

\section{ACKNOWLEDGEMENTS}

The authors thank the members of the SI,C Damping Ring group for helpful discussions.

\section{REFERENCES}

[1] P. Krejcik, el al., Proc. of the 1993 IEEE Particle Acc. Conf., Washington D.C., 1993, p. 3240.

[2] K. Bane, et al., WAC17, this conference.

[3] K. Bare and K. Oide. Proc. of the 1993 IF.EF. Parlicle Acc. Cons., Washington D.C., 1993, p. 3339.

[4] M. Minty, private communication.

[5] K. Oide, KEK-PREPHINT-94-138, Nov 1994

[6] A. Chao, et al, WABIU, this conference.

[7] B. Chen, WAC31, this conference.

[8] K. Bane and C.K. Ng. Proc. of the 1993 [EEE Particle Acc. Conf., Washington D.C. 1993, p. 3432.

[9] F. Ebeling, et al, MAF]A Users Guide. 1992.

[10] J. Haissinski, /I Nuovo Cimento, 18B, No. 1, 7ะ (1973).

[Il] See e.g. R. Siemann, Nrol, /ngtr Heth. 203, 57 (1952).

[12] $K$. Oide and $k$. Yokoya, KE.h-Preprint-90-10. 1990.

[13] A. Chao, Physics of Collective Beam Instabuhtues in High Energy Accelerators, [1. Wiley \& Sons. New York. 1!193]. 


\section{DISCLAIMER}

This repor was prepared as an account of work sponsored by an agency of the United States Goveroment. Neither the United Stales Government nor any agency thereof, nor any of their employees, makes any warranty, express or implied, or assumes any legal liabilucy or responsibility for the accuracy, completeness, or usefulness of any information, apparalus, product, or process disclosed, or represents that its use would not infringe privately owned rights. Reference herein to any specific commercial product, process, or service by trade name, Lsademark manufactures, or otherwise does not necessarily constitute or imply its endorsemed, recommendation. or favoring by the United States Government or any agensy thereof. The views and opinions of authors expressed herein do not necessarily state or reflect those of the United States Government or any agency thereof. 